\title{
Optical continuum properties of the NLS1 galaxy Mrk 335 in 1995-2004^
}

\author{
V. T. Doroshenko ${ }^{1,3}$, S. G. Sergeev ${ }^{2,3}$, N. I. Merkulova ${ }^{2,3, \star \star}$, E. A. Sergeeva ${ }^{2,3}$, and Yu. V. Golubinsky ${ }^{2,3}$ \\ ${ }^{1}$ Crimean Laboratory of the Sternberg Astronomical Institute, University of Moscow, Russia; p/o Nauchny, \\ 98409 Crimea, Ukraine \\ e-mail: doroshen@sai.crimea.ua \\ 2 Crimean Astrophysical Observatory, p/o Nauchny, 98409 Crimea, Ukraine \\ e-mail: [sergeev; selena;urix] @crao.crimea.ua \\ 3 Isaak Newton Institute of Chile, Crimean Branch
}

Received 2 November 2004 / Accepted 8 March 2005

\begin{abstract}
We present results of UBVRI photometry of Mrk 335 in 1995-2004. This object has a large amplitude of variability, reaching about 1.1, 0.9, $0.7 \mathrm{mag}$ in the $U, B, V$ and $0.3 \mathrm{mag}$ in the $R \mathrm{c}, I \mathrm{c}$ bands, respectively. This photomerical behaviour as well as the spectral energy distribution in the optical part of the spectrum is typical of Seyfert 1 galaxies. The structure function $(S F)$ analysis of Mrk 335 has shown that the variability of Mrk 335 can be caused by stochastic superposition of independent flares in a wide interval of durations, the longest of which has a duration of about several hundred days. The slope of the power-law portion of the $S F b \sim 0.6-0.7$ for the time scale from several days to several hundreds of days. It is close to the estimates for disk instability models. The estimate of the extension of the instability zone in an accretion disk (about $300 R_{\mathrm{S}}$ ) is also evidence that the instability in an accretion disk is able to explain the long-term optical variability. A cross-correlation function $(\mathrm{CCF})$ shows some evidence that the delay of fluxes in the $V, R, I$ bands relative to the $U$ band can be of some days, and there is a tendency for lags to be systematically higher for the $R, I$ bands than for $B, V$.
\end{abstract}

Key words. galaxies: active - galaxies: nuclei - galaxies: Seyfert - galaxies: individual: Mrk 335 - galaxies: photometry

\section{Introduction}

Mrk 335 is a nearby Seyfert galaxy at a redshift $z=0.026$. According to Veron-Cetty et al. (2001), Mrk 335 belongs to a class of NLS1 galaxies. This subclass of AGNs was identified by Osterbrock \& Pogge (1985) by their optical emissionline properties: they have rather strong high-ionization lines, strong optical Fe II emission lines, $F W H M(\mathrm{H} \beta)$ is less than $2000 \mathrm{~km} \mathrm{~s}^{-1}$ and the intensity ratio of lines [OIII] $\lambda 5007$ to $\mathrm{H} \beta$ is less than 3 . Their $\mathrm{H} \beta$ profiles have a tendency to exibit a Lorentzian profile rather than a Gaussian profile. These profiles also tend to have a blue asymmetry in the $\mathrm{H} \beta$ line. NLS1 sources have strong infrared emission, however, they are seldom radio-loud objects. Later it was discovered that, as a rule, NLS1s have unusually rapid and high-amplitude X-ray variability (Boller et al. 1996)

Indeed, Mrk 335 has the intensity ratio of lines Fe II to $\mathrm{H} \beta$ of about 0.62 and $\mathrm{H} \beta F W H M=1640 \mathrm{~km} \mathrm{~s}^{-1}$, and also relatively strong coronal lines. Using UV, optical and IR ob-

^ Full Tables 1 and 2 are only available in electronic form at the CDS via anonymous ftp

to cdsarc.u-strasbg.fr $(130.79 .128 .5)$ or via

http://cdsweb.u-strasbg.fr/cgi-bin/qcat?]/A+A/437/87

$\star \star$ Deceased December 12, 2004. servations, Edelson \& Malkan (1986) have constructed the broad-band spectral energy distribution in Mrk 335 which includes 6 components: an infrared power law, the near-infrared bump, an ultraviolet black-body component with a temperature $25600 \mathrm{~K}$, the galactic star light, and the Balmer and the Pashen continua. Many researchers reported variability of Mrk 335 in the X-ray region $(2-10 \mathrm{keV})$ on time scale of hours and days (Turner \& Pounds 1988; Bianchi et al. 1999; Gondoin et al. 2002). Simultaneous X-ray, UV and optical spectral observations in December 1990 allowed some astronomers to study not only an emission-line spectrum in the range of $\lambda \lambda$ 912-8410 $\AA$, but also to obtain the spectral energy distribution (SED) of Mrk 335 up to $10 \mathrm{Kev}$ (Turner et al. 1993; Zheng et al. 1995). It was shown that the X-ray spectrum could be a high energy tail of the accretion disk spectrum, which provides a good match to the UV and optical continuum of Mrk 335. A time delay of 16.8 days between the $\mathrm{H} \beta$ emission line and the continuum variations was found from observations of Mrk 335 in 1989-1996 (Kassebaum et al. 1997; Peterson et al. 1998a).

Unfortunately, there is no systematic broad band photometric monitoring of this galaxy. In this paper we present the results of UBVRcIc observations of Mrk 335 made in Crimea in 1995-2004. 
Table 1. The observed $U B V$ magnitudes of Mrk 335 through the aperture $A=27.5^{\prime \prime}$.

\begin{tabular}{lcccccc}
\hline \hline $\begin{array}{l}\text { JD } \\
2450000+\end{array}$ & $U$ & $\sigma U$ & $B$ & $\sigma B$ & $V$ & $\sigma V$ \\
\hline 0004.258 & 13.117 & 0.020 & 14.176 & 0.016 & 13.938 & 0.023 \\
0006.354 & 13.154 & 0.020 & 14.167 & 0.016 & 13.937 & 0.016 \\
0008.268 & 13.129 & 0.016 & 14.134 & 0.013 & 13.869 & 0.017 \\
$\ldots$ & $\ldots$ & $\ldots$ & $\ldots$ & $\ldots$ & $\ldots$ & $\ldots$ \\
2973.351 & 13.619 & 0.016 & 14.595 & 0.016 & 14.221 & 0.020 \\
2996.305 & 13.912 & 0.032 & 14.736 & 0.018 & 14.317 & 0.027 \\
2997.313 & 13.866 & 0.024 & 14.781 & 0.030 & 14.268 & 0.030 \\
\hline
\end{tabular}

\section{Observations}

Photoelectric monitoring of Mrk 335 in the $U B V$ bands in 1995-2004 was carried out at the Crimean Laboratory of the Sternberg Astronomical Institute of Moscow University. A photometer with a photomultiplier detector is attached to the 60-cm Zeiss telescope. The instrumental system with the broad-band filters used in this photometer is close to Johnson's standard photometric system. More details of the $U B V$ photometer can be found in Lyuty (1971). The observations were made through an aperture $A=27.5^{\prime \prime}$ in diameter. The star with coordinates $\alpha=00^{\mathrm{h}} 06^{\mathrm{m}} 26^{\mathrm{s}}, \delta=+20^{\circ} 10^{\prime} 18^{\prime \prime}$ (2000) was chosen as a standard. The magnitudes of this comparison star were obtained by us: $U=11.478 \pm 0.010, B=11.463 \pm 0.009$, and $V=10.956 \pm 0.009$. There are $231 U B V$ observations of Mrk 335 which cover about 3000 nights. The mean and median intervals for the photoelectric observations are equal to 10 and 2 days, respectively. Typical uncertainties of the observations are about $0.030,0.018$ and $0.014 \mathrm{mag}$ in the $U, B, V$ bands. The observed $U B V$ magnitudes of Mrk 335 with their uncertainties are given in Table $1^{1}$. We have observed Seyfert galaxies in the $B, V, R, I$ bands since the end of 2001 using the 70-cm telescope (AZT-8) of the Crimean Astrophysical Observatory equipped with an AP7p CCD camera. The CCD camera is mounted at the prime focus $(f=$ $282 \mathrm{~cm}$ ) of the telescope. The CCD array has a size of $512 \times 512$ pixels giving the scale $1.755^{\prime \prime} /$ pixel and a field of view of $15^{\prime} \times 15^{\prime}$. As a rule, for each object we obtained not less than four images per night in each filter, preceded and followed by one bias frame and two dark frames. The flat fields were taken using the dawn and dusk sky. The unbiased, dark-subtracted and flat-fielded images were then measured. Photometric measurements and reductions were carried out by aperture photometry using the photometry packages developed by S. G. Sergeev. The aperture diameter was set to $15^{\prime \prime}$. The sky background was determined in a ring around each object. More details on the observational method, error estimation, data reduction and selection of comparison stars in Seyfert Galaxy fields can be found in Doroshenko et al. (2005). For Mrk 335 we analyzed 231 images obtained during the 67 nights from 5 January 2002 to 17 January 2004. The treatment of Mrk 335 was carried out relative to star No. 4 with coordinates $\alpha=00^{\mathrm{h}} 06^{\mathrm{m}} 20.2^{\mathrm{s}}$ and $\delta=+20^{\circ} 10^{\prime} 50.5^{\prime \prime}$ (2000)

\footnotetext{
${ }_{1}$ The full table is available in electronic form at the CDS.
}

Table 2. The observed BVRcIc magnitudes of Mrk 335 through $A=$ $15^{\prime \prime 2}$.

\begin{tabular}{ccccccccc}
\hline \hline $\begin{array}{c}\mathrm{JD} \\
2450000+\end{array}$ & $B$ & $\sigma B$ & $V$ & $\sigma V$ & $R \mathrm{c}$ & $\sigma R \mathrm{c}$ & $I \mathrm{c}$ & $\sigma I \mathrm{c}$ \\
\hline 2280.16193 & 14.233 & 0.006 & 14.043 & 0.005 & 13.602 & 0.005 & 13.327 & 0.005 \\
2300.19097 & 14.234 & 0.015 & 14.010 & 0.009 & 13.585 & 0.006 & 13.295 & 0.007 \\
2309.21102 & 14.275 & 0.025 & 14.026 & 0.016 & 13.603 & 0.012 & 13.304 & 0.022 \\
$\ldots$ & $\ldots$ & $\ldots$ & $\ldots$ & $\ldots$ & $\ldots$ & $\ldots$ & $\ldots$ & $\ldots$ \\
3004.27434 & 14.724 & 0.011 & 14.398 & 0.009 & 13.925 & 0.006 & 13.628 & 0.007 \\
3007.22611 & 14.665 & 0.014 & 14.387 & 0.009 & 13.922 & 0.006 & 13.604 & 0.008 \\
3022.26464 & 14.661 & 0.007 & 14.338 & 0.006 & 13.891 & 0.005 & 13.589 & 0.006 \\
\hline
\end{tabular}

not far from the galaxy. Our calibration of star No. 4 was based on the secondary standard stars in fields of other AGNs taken mainly from González-Pérez et al. (2001): 3C 66 A (7 common nights), S5 0716+71 (8 nights), OJ 287 (8 nights), BL Lac (19 nights), Akn 564 (27 nights), NGC 7469 (27 nights) and NGC 7603 (22 nights). Only good photometric nights were chosen for calibration, when the root mean square changes of the star fluxes were found to be less than $1.5-2 \%$. The colour transformation equations were taken into account. Finally, we calculated the unweighted mean value over these different calibrations. The magnitudes of this star were computed: $B=$ $15.406 \pm 0.010, V=14.251 \pm 0.009, R \mathrm{c}=13.618 \pm 0.005$ and $I c=13.050 \pm 0.006$. The magnitudes for star 4 that we determined are in good agreement with the previously calculated magnitudes of Bachev et al. (2000) in the $V$ and $R \mathrm{c}$ bands, but slightly differ in the Ic filter. However, the disagreement in the Ic band is well within experimental error. The mean and median intervals for recent CCD observations are equal to 11 and 2 days, respectively. Typical uncertainties of the Mrk 335 CCD observations are about of 0.014, 0.009, 0.006 and $0.007 \mathrm{mag}$ in the $B, V, R \mathrm{c}, I \mathrm{c}$ bands. The observed $B, V, R c, I c$ magnitudes of the Mrk 335 nucleus with their uncertainties are given in Table $2^{2}$. In order to determine a relation between $C C D$ and photoelectric $B, V$ magnitudes, i.e. to determine the contribution of the host galaxy in the ring $15^{\prime \prime}-27.5^{\prime \prime}$, we also processed some CCD images of Mrk 335 with a circular aperture of $27.5^{\prime \prime}$ in diameter. For this purpose we used the nights when the Mrk 335 nucleus was in a state of low brightness, and when the seeing was less $5^{\prime \prime}$, i.e. the contribution of the nucleus to the total brightness in the aperture of $15^{\prime \prime}$ diameter was small. On average, for three selected nights satisfying the conditions we obtained that the brightness of the galaxy in the ring between $A=27.5^{\prime \prime}$ and $A=15^{\prime \prime}$ is (in units $10^{-16} \mathrm{erg} \mathrm{cm}^{-2} \mathrm{~s}^{-1} \AA^{-1}$ ) $1.300,5.232,3.800$ and 3.433 in the $B, V, R \mathrm{c}, I \mathrm{c}$ filters, respectively, and in stellar magnitudes are $B=19.3 \pm 0.9, V=17.10 \pm 0.15, R \mathrm{c}=16.93 \pm 0.07$, $I c=16.38 \pm 0.01$. Adding the calculated fluxes in the ring to our Mrk 335 fluxes measured in the aperture $A=15^{\prime \prime}$, we recalculated the fluxes and magnitudes in the $B, V, R, I$ bands to the aperture $A=27.5^{\prime \prime}$. After that, using the overlapping interval of observations, we fitted an ordinary $y \mid x$ unweighted regression the $B, V$ magnitudes of Mrk 335 in $A=27.5^{\prime \prime}$ from the $\mathrm{CCD}$ to the photoelectric $B, V$ magnitudes.

2 The full table is available in electronic form at the CDS. 


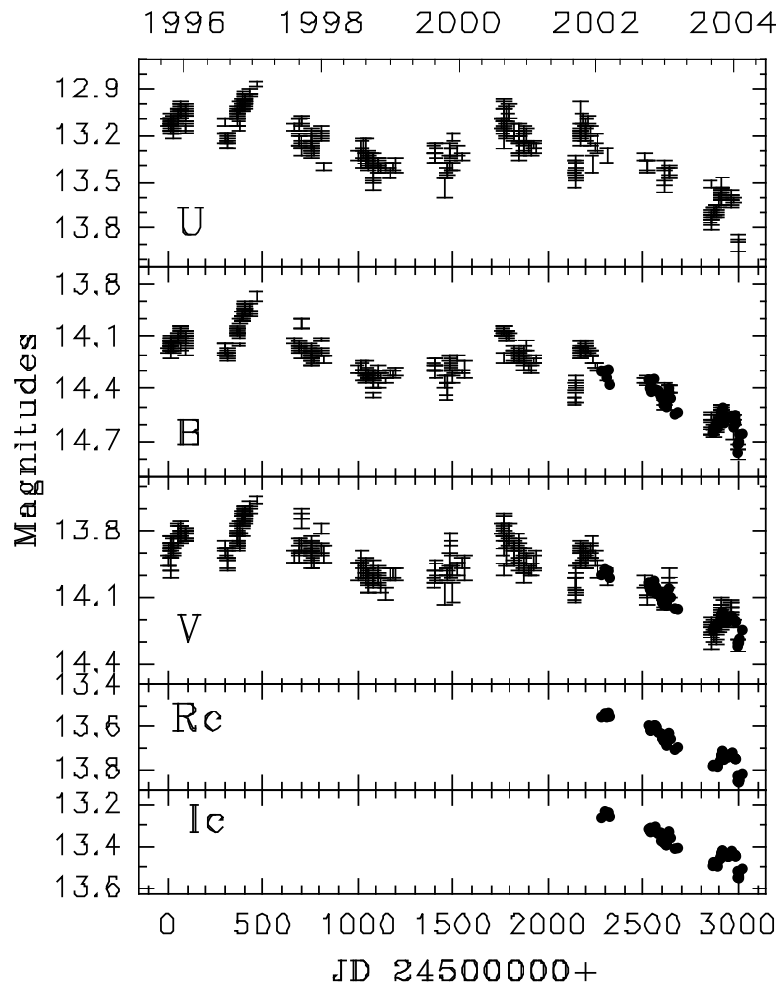

Fig. 1. $U, B, V, R \mathrm{c}, I \mathrm{c}$ light curves for Mrk 335.

\section{Light curves}

Light curves for Mrk 335 in $A=27.5^{\prime \prime}$ are plotted in Fig. 1 where by vertical bars we display $\pm 1 \sigma$ observational errors for photoelectric data, and by filled circles we show the CCD data. In the latter case the observational errors are less than the symbol size. In 1995-2004 the brightness decreased systematically, and variations different amplitudes and durations are present. The main parameters of variability are shown in Table 3 where fluxes and rms are given in units of mJy. The amplitude of variability in the $U$ band is maximal, and of more than $1 \mathrm{mag}$. Such amplitude variability is typical for Sy1 galaxies. We mentioned in the Introduction that in 1989-1996 long-term spectral monitoring of Mrk 335 was carried out (Kassebaum et al. 1997; Peterson et al. 1998a). Using the overlapping time interval between the spectral continuum data near $\lambda 5100 \AA$ and our photometric observations with the $V$ filter in 1996-1997, we could obtain a more complete light curve in the $V$ band for the time interval from 1989 to 2004 . The composite light curve is shown in Fig. 2 where magnitudes obtained from $\lambda 5100 \AA$ fluxes are displayed by thick bars. The others symbols are the same as in Fig. 1. One can see in Fig. 2 that the spectral and photometric data supplement each other very well. The Mrk 335 variability in the large time interval is equally complex. The amplitude of the fast component in 1989-1995 is almost the same as in 1995-2004, while the amplitude of the slow component has a large variation, up to a factor of 2.6, noticeably larger than the measurement errors of $0.5-2.7 \%$.
Table 3. Variability statistics.

\begin{tabular}{lcccccc}
\hline \hline Band & $F_{\text {mean }}$ & $\sigma_{\text {obs }}$ & $\sigma_{\text {err }}$ & $\sigma_{\text {int }}$ & $F_{\text {var }}$ & $R_{\max }$ \\
\hline Uphe & 9.076 & 1.631 & 0.245 & 1.612 & 0.178 & 2.621 \\
Bphe & 8.649 & 1.384 & 0.140 & 1.377 & 0.159 & 2.319 \\
Vphe & 9.717 & 1.236 & 0.232 & 1.214 & 0.125 & 1.835 \\
Bccd & 6.719 & 0.682 & 0.085 & 0.677 & 0.101 & 1.550 \\
Vccd & 7.996 & 0.631 & 0.068 & 0.627 & 0.078 & 1.384 \\
Rccd & 10.258 & 0.797 & 0.065 & 0.794 & 0.077 & 1.358 \\
Iccd & 11.146 & 0.795 & 0.088 & 0.790 & 0.071 & 1.343 \\
\hline
\end{tabular}

Notes: $\sigma_{\text {int }}=\sigma_{\text {intrinsic }} ; \sigma_{\text {int }}^{2}=\sigma_{\mathrm{obs}}^{2}-\sigma_{\mathrm{err}}^{2} ; F_{\mathrm{var}}=\sigma_{\text {imnt }} / F_{\text {mean }} ; R_{\max }=$ $F_{\max } / F_{\min } . F=$ Flux in mJy.

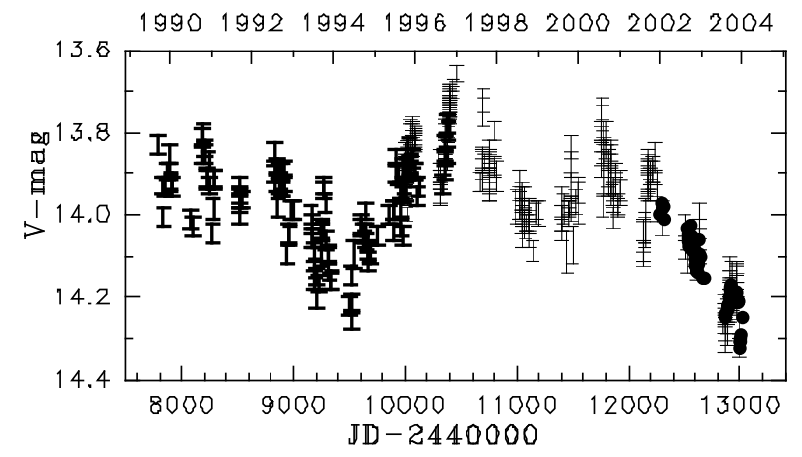

Fig. 2. Light curve in $V$ for Mrk 335 combined from spectral continuum data near $\lambda 5100 \AA$ - thick bars, and photometric $V$ data - thin bars (photoelectric magnitudes) and filled circles (CCD data).

\section{Spectral energy distribution of the variable source in Mrk 335}

We suggested that the shape of the nuclear spectral energy distribution remains constant in spite of variations in flux. In this case there is an exact linear relationship between fluxes from different spectral bands in "flux - flux" diagrams. The slope of the regression line determines the observed color index of the variable component. There is a high correlation between the two fluxes under consideration. We determined the next observed color indices of the variable component (correlation coefficient $\boldsymbol{r}$ is given in parentheses): $U-B=-1.064 \pm 0.017$ $(\boldsymbol{r}=0.972) ; B-V=0.092 \pm 0.018(\boldsymbol{r}=0.967) ; V-R \mathrm{c}=$ $0.449 \pm 0.023(\boldsymbol{r}=0.989)$ and $V-I \mathrm{c}=0.653 \pm 0.022$ $(\boldsymbol{r}=0.987)$. The $U-B$ and $B-V$ color indices are close to the intrinsic color indices obtained by Winkler (1997) for a typical Syl galaxy: $U-B=-1.10 \pm 0.04, B-V=0.00 \pm 0.03$, $V-R \mathrm{c}=0.20 \pm 0.08$ and $V-I \mathrm{c}=0.35 \pm 0.10$. This means that the extinction in the direction of Mrk 335 is small. According to Veron-Cetty et al. (2001), the Galactic hydrogen column density for Mrk 335 equals $3.8 \times 10^{20} \mathrm{~cm}^{-2}$. Using the relation $N(\mathrm{H}) / E(B-V)=5.8 \times 10^{21}$ (Bohlin et al. 1978) and $A(V) / E(B-V)=3$, we obtain $A(V)=0.19$ mag. Figure 3 shows the dereddened spectral energy distribution (SED), normalized to the $V$ band. It is plotted by a thick line for Mrk 335 and by a dashed line for a typical Sy1 galaxy. Comparing the two SEDs, we can see a small excess for Mrk 335 in the $U$ and in the $R \mathrm{c}, I \mathrm{c}$ bands also. 


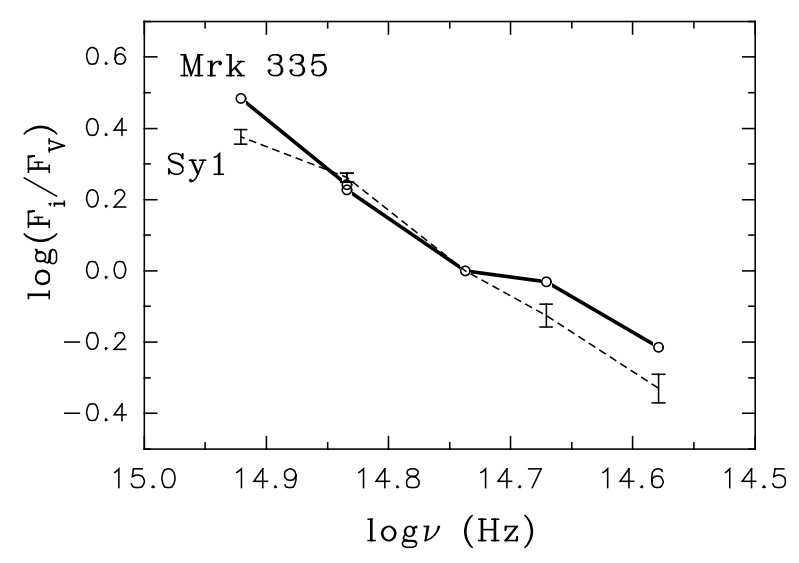

Fig. 3. The spectral energy distribution in the variable source of Mrk 335.

\section{Structure function}

For the analysis of our time series we applied the structure function $(S F)$ technique. The first structure function was introduced by Kolmogorov in 1941 for analysis of the statistical problems of turbulence theory (Kolmogorov 1941). The main properties of the $S F$ have been described in many papers and now $S F$ analysis is widely used for the study of time series of AGNs (e.g. Kawaguchi et al. 1998; Cid Fernandes et al. 2000; Collier \& Peterson 2001). The time scale and amplitude of variability as well as the slope of the power-law portion of the $S F$, which depends on the nature of the intrinsic variation of the source, are the key parameters of the $S F$.

\subsection{Observed SFs}

We computed the $S F$ s for Mrk 335 using our CCD and photoelectric observations in the $U, B, V$ filters, as well as combined spectral and photometric $V$ data. The $S F$ s are plotted by points in Fig. 4. We use logarithmic time bins for the calculation of the $S F$.

Three features can be observed in these $S F$ s. The first feature is the presence of a low plateau on short time scales. This part of the $S F$ is due to the white noise introduced by the flux measurement errors. The second feature is a linear part of the relation " $\log S F-\log \tau$ " over a wide range of time scales. The time of the beginning and end of the powerlaw portion of the $S F\left(\log \tau_{\min }\right.$ and $\log \tau_{\max }$, respectively) was determined by visual inspection. The logarithmic slopes (b) of the $S F$ s for different filters are very similar (Fig. 4 and Table 4). The time of reaching the upper plateau is the third feature of the $S F$. We see that this time cannot be estimated reliably in $U B V$. However the $S F$ of the full $\mathrm{V}$ data set over the wide time interval (1989-2004), plotted on the lower panel of Fig. 4, displays this plateau more clearly. The value $\tau_{\max }$, obtained by visual inspection, is to about 500-600 days. The thick line in Fig. 4 represents the function $f(t)=2 \sigma_{\text {err }}^{2}+a \cdot t^{b}$, where $\sigma_{\text {err }}$ is the measurement error taken from photometric observations, while parameters $a$ and $b$ correspond to the best fit of the observed $S F$ s. Indeed, at least 4 long-term structures with durations of about 360-640 days

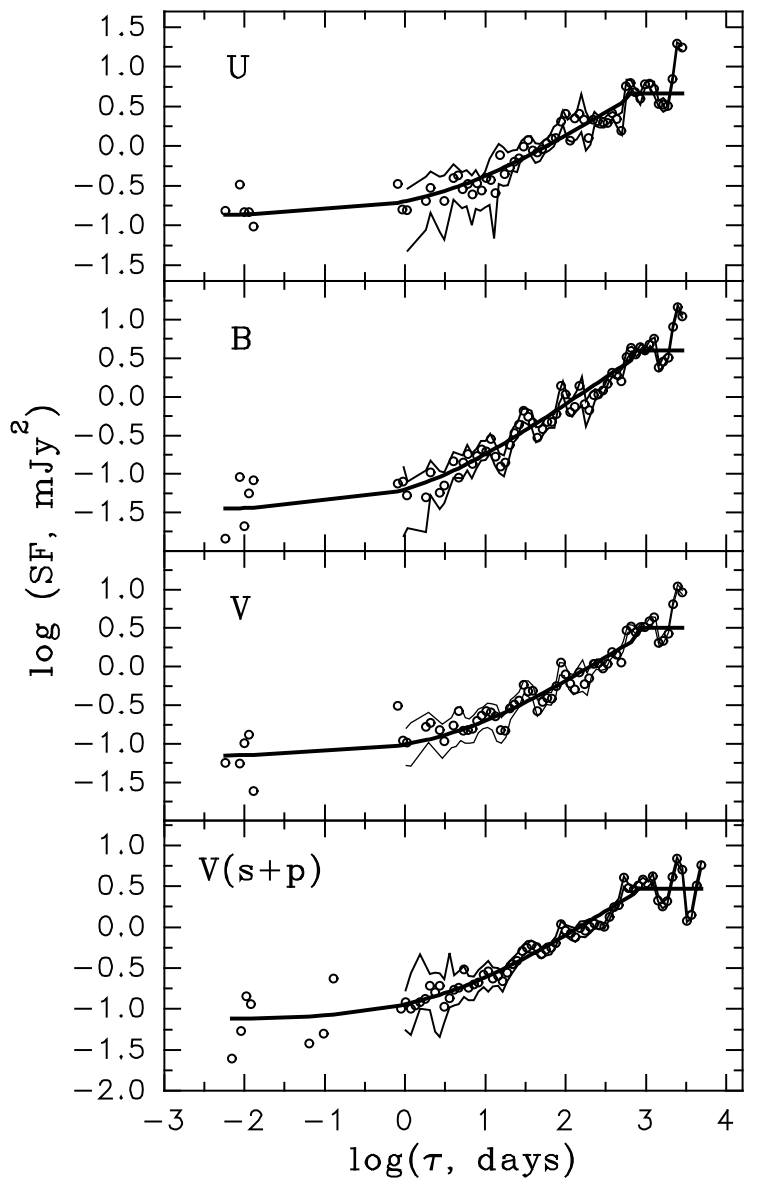

Fig. 4. The structure function in the $U, B, V$ filters for Mrk 335 based on our photoelectric and CCD observations (three upper panels) and based on combined spectral and photometric $V$ data shown in the lower panel of Fig. 2. The thick line describes the function $f(t)=$ $2 \sigma_{\text {err }}^{2}+a \cdot t^{b}$, where $\sigma_{\text {err }}$ is taken from photometric observations and parameters $a$ and $b$ correspond to the best fitting of the observed $S F \mathrm{~s}$. The two thin lines show $\pm 1 \sigma$ uncertainties of the $S F$ calculated with the FR/RSS technique.

are seen in the combined light curve of Mrk 335 in the next time intervals: JD 2448 510-8950; 2450 310-2450 670; 2451 500-2452 140; 2452 140-2 452520 (Fig. 1). As we can see in Fig. 1, the light curves from 1995 to 2004 demonstrate a gradual decrease of brightness. This leads to the steepening of the structure function on the longest time scale because of the linear trend resulting in $S F \propto \tau^{2}$.

\subsection{Uncertainties of the SFs}

The uncertainties of the observed $S F$ s are assumed to come from the limited statistics of data pairs in bins, from the flux measurement errors and from the observational sampling of the light curves. Because the number of pairs of data points in the bin can be very large when $\tau$ is large, the first type of errors dominates in the part of the $S F$ where $\tau$ is very small. The second type of uncertainty can be assessed by modifying the flux of the light curve by random Gaussian deviations based on the quoted error for each data point, known as the flux randomization (FR) method. The third type of uncertainty can be 
Table 4. Some parameters of the $S F$ s for Mrk 335.

\begin{tabular}{lccccccc}
\hline \hline Band & $N$ points & Time & $\sigma_{\text {err }}$ & $b_{\text {mean }} \pm 1 \sigma$ interval & $\tau_{\max }$ & Plato \\
& of light & interval & & $0.3<\log \tau<2.75$ & & $2 \sigma_{\text {pro }}^{2}$ \\
\cline { 2 - 8 } & curves & days & mJy & \multicolumn{2}{c}{ where $\tau$ in days } & days & $\mathrm{mJy}^{2}$ \\
\hline$U$ & 226 & 2993 & 0.260 & 0.72 & $0.61-0.98$ & 560 & 0.68 \\
$B$ & 296 & 3018 & 0.207 & 0.71 & $0.61-0.72$ & 560 & 0.60 \\
$V$ & 296 & 3018 & 0.208 & 0.65 & $0.52-0.74$ & 560 & 0.46 \\
$V_{s+p}$ & 419 & 5234 & 0.193 & 0.63 & $0.53-0.73$ & 560 & 0.46 \\
\hline
\end{tabular}

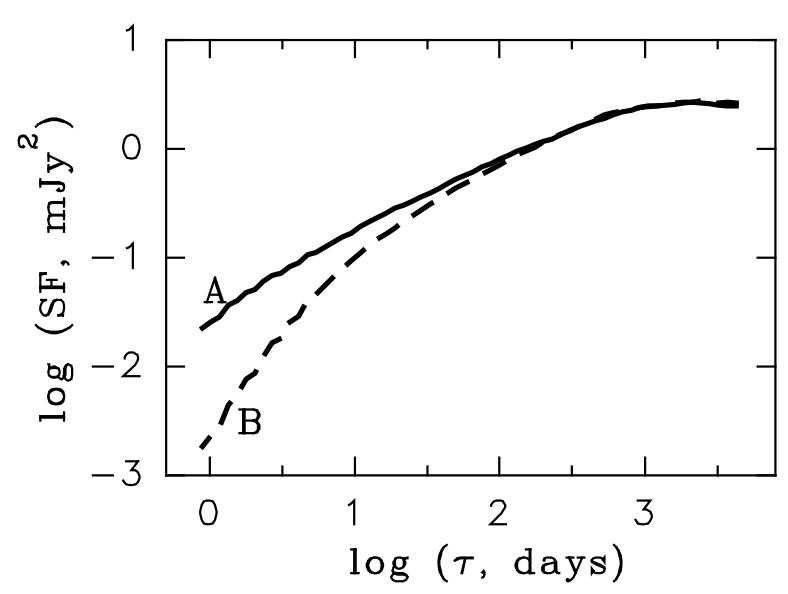

Fig. 5. The patterns of two mean structure functions performed for 400 Monte-Carlo simulations of light curves formed by superposition of independent flares with Gaussian profiles and a wide range of flare life-times: $0.2-560$ days (solid line A) and with a shorter range of flare life-times (dashed line B): 2-560 days with the same parameters $\alpha$ and $\beta$ (see text).

estimated with the bootstrep method or the random subset selection (RSS) method. In this method, from the original $N$ data points we can take a subset containing $N$ randomly selected points without regard to whether or not they have been previously selected. In such a subset some data points are repeated more than once, and some of the original points are ignored. The multiple points are removed, so the size of the subset is reduced. For each realization of the light curve through the flux randomization (FR) and random subset selection (RSS) the $S F$ was computed in the same manner as was done for the observed light curve. Computing a large number of independent realizations of light curves, we calculate the mean $S F$ and the standard deviation (rms) from the mean $S F$. This FR/RSS method was used by Peterson et al. (1998b). Then the $\pm 1 \sigma$ interval of uncertainties on the power-law slope can be computed from the slope of the $($ mean $+\mathrm{rms}) S F$ and from the the slope of the (mean-rms) $S F$ (see column six in Table 4). In Fig. 4 two thin lines display $\pm 1 \sigma$ uncertainties of the $S F$ computed with the FR/RSS technique.

\subsection{Poissonian model}

The jaggedness of the light curves has prompted researchers to consider them as the superposition of independent flares randomly distributed in time with a wide range of amplitudes and durations. This is the so-called a Poissonian model for
AGN variability. Sergeev (1999) in his thesis showed that if the flare amplitude, $A(\omega)$, depends on $\omega$ as a power law function, $A(\omega) \propto \omega^{\alpha}$, and if the number of flares $n$ per unit time with duration $\omega$ is given by a power law distribution, $n(\omega) \propto \omega^{\beta}$, then the $S F$ asymptotically tends to a power law shape, $S F(\tau) \propto \tau^{b}$, where $b=\alpha+2 \beta+2$. This power law is however valid only for the the time interval $\tau \gg \omega_{\min }$ and $\tau \ll \omega_{\max }$, where $\omega_{\min }$ and $\omega_{\max }$ is the minimal and maximal duration of flares. Here we considered flares with the Gaussian profiles. We adopted that the $\omega$ value is the 0.7 level of the profile maximum. It was shown by Sergeev (1999) and also by Cid Fernandes et al. (2000) that the Poissonian analysis is insensitive to the shape of flares. We have performed Monte-Carlo simulations with the following parameters: $\Delta \mathrm{T}-$ the mean time interval between two sequential flares, $T_{\min }$ and $T_{\max }$ - the minimal and maximal duration of flares and the parameters $\alpha$ and $\beta$, by relation $b=\alpha+2 \beta+2$, where $b$ is to the logarithmic slope of the observed $S F$. From the slope of the $S F$ it is impossible to obtain separately the values of the $\alpha$ and $\beta$ parameters, but it is possible to put some restrictions on the parameters using rational assumptions based on physical considerations and common sense. The number of flares is larger for low amplitudes and for short durations. This means that $\alpha$ must be negative, but $\beta$ must be positive. Note that the $T_{\max }$ of flares cannot be more than the time of the beginning of the upper plateau in the observed SF. We performed 400 Monte-Carlo simulations of light curves from a superposition of independent flares with Gaussian profiles and with various durations and amplitudes. We then computed the $S F$ for each simulated light curve in the same way as for the observed light curve and obtained the mean SF. The Monte-Carlo simulations showed that only stochastic process with flares in a wide range of durations provides a good fit to the observed $S F$. In Fig. 5 we show two mean $S F$ s resulting from such simulations. One of them (A), plotted with a solid line, was calculated with $\alpha=-2.43, \beta=0.5, \Delta T=0.01$ days and for the flare life-times from $T_{\min }=0.2$ days to $T_{\max }=560$ days. Another $S F(\mathrm{~B})$, plotted with the dashed line, was calculated with the same parameters $\alpha, \beta$ and $T_{\max }$, but $T_{\min }=2$ days, i.e. 10 times larger. The time interval between the sequential flares was increased by 18 times ( $\Delta T=0.18$ days $)$ in order to obtain the same observed variance. A comparison shows that the decreasing the possible range of flare life-times makes the $S F$ steeper in the small time intervals. In Fig. 6 we show the observed structure function (open circles) in the $V$ filter for the combined light curve plotted in Fig. 2, and the mean $S F$ (thick line) for multiple simulated light curves calculated for the Poissonian model with parameters that correspond to line A in Fig. 5. Thus, that the superposition of independent flares over a wide interval of durations gives quite satisfactory estimates of the variability parameters for Mrk 335.

\subsection{Discussion of the SF}

The consideration of AGN long-term variability in the context of a Poissinian model encompasses a large class of physical models which claim to explain the nature of their variability. 


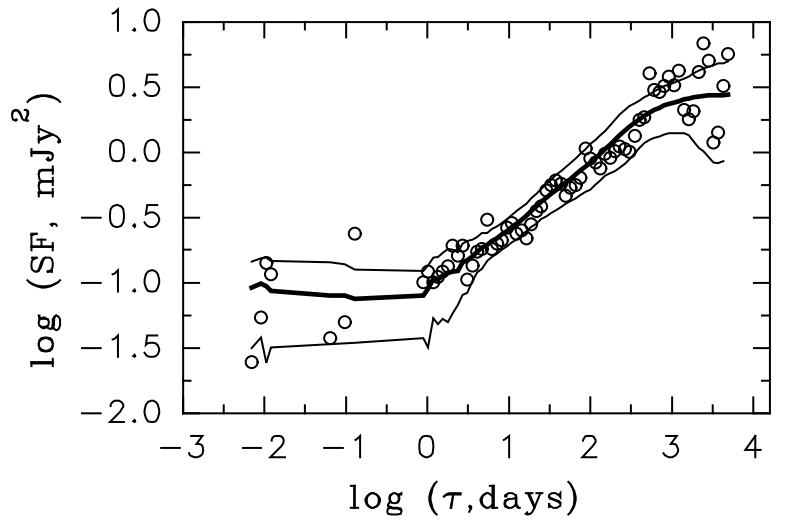

Fig. 6. Comparison of the observed structure function for Mrk 335 in the $V$ filter based on the spectral and photometric $V$ data (open circles) with the mean $S F$ (thick line) performed for 400 Monte-Carlo simulated light curves corresponding to superposition of flares with different durations and amplitudes. The parameters for simulations were taken as follows: $\alpha=-2.43, \beta=0.5, \Delta T=0.01$ days, $T_{\min }=0.2$ days and $T_{\max }=560$ days. $2 \sigma_{\text {err }}^{2}$, where $\sigma_{\text {err }}$ corresponds to the observed measurement errors, was added to the mean simulated $S F$. The two thin lines show $\pm 1 \sigma$ interval of the simulated $S F$ s.

One sort of model, which does not always involve a black hole in the centre of galaxy, is the starburst (SB) model (see, e.g. Aretxaga \& Terlevich 1994). In this case the energy is generated by violent star formation activity in the innermost regions of AGNs. Then flux variability is associated with evolution of massive stars, supernovae and supernova remnants. Another set of models is related to the existence of a supermassive black hole and surrounding accretion disk in AGN. Occasional flare events are caused by accretion disk instabilities of several kinds. This group includes disk-instability (DI) models (see, e.g. Haardt et al. 1994; Kawaguchi et al. 1998) or relativistic stellar collisions in dense clusters surrounding a massive black hole (Courvoisier et al. 1996), and many other models. A detailed discussion about the implications of the above-mentioned variability parameter estimates, such as amplitude, minimal and maximal time of variability or slope of the power-law portion of the $S F$ for different scenarios for AGN variability is beyond the scope of this work. We would like only to note that for simulated light curves in the SB model the logarithmic slope $b$ of the $S F\left(S F \propto \tau^{b}\right)$ is about 1.5-1.8 and $b \sim 0.8-1.0$ in the DI model, according to Kawaguchi et al. (1998). The observed light curve of Mrk 335 has $b \sim 0.6-0.7$ which is more consistent with the estimates for disk instability models.

The time $T_{\max }$, when the $S F$ reaches the upper plateau, is a robust, nevertheless, physically meaningful characteristic time scale for each source. In SB models this time may be connected to the life time of supernova remnants. For DI models $T_{\max }$ can possibly be attributed to accretion disk time scales. There are a number of physical time scales associated with sources and they depend on the mass of the black hole, the emission distance from the center, and the properties of the accretion disk such as the disk viscosity parameter $\gamma$ and the disk thickness, which in turn depends on the accretion rate. If the black hole mass of Mrk 335 is equal to $4.8 \times 10^{6} M_{\odot}$ (Wandel et al. 1999) and if we assume that the thermal time scale is 560 days, then $\left(r / 3 R_{\mathrm{S}}\right)^{3 / 2}=10^{4} \times \gamma_{0.1}$ (Czerny 2005). Here $\gamma_{0.1}$ is the standard viscosity parameter in units of 0.1 . The real value of the viscosity parameter $\gamma$ is unknown. Usually it is assumed to be $\gamma \sim 0.01$. Then we have $r \approx 300 R_{\mathrm{S}}$ for the size of the thermally unstable zone of the accretion disk. Taking into account many uncertain factors we can conclude that this is a quite reasonable estimate. For example, Czerny et al. (1999) have shown that the instability zone in AGNs can start at $200 R_{\mathrm{S}}$. Thus, the disk instability can be a source of the observed long term optical variability of AGNs.

\section{Lags between variations in the $U$ and the $B, V$, Rc, Ic bands}

To understand the processes occuring in galactic nuclei it is very important to know which changes are primary and which are secondary. Several models give different predictions about the sign and value of the lag between different spectral bands. Many authors have considered models where the X-ray source is located above the accretion disk, illuminating it. X-ray radiation is re-processed by the accretion disk. A re-processing geometry naturally accounts for the existence of a lag because colder parts of the re-processing zone are located farther from the hard X-ray source.

Below we discuss the time relationship between flux variations in different spectral regions of the optical continuum in the observations. We performed a cross-correlation analysis using an interpolation cross-correlation function which we applied to our data of Mrk 335 in 2001-2004. We computed the delays between fluxes in the $U$ filter and fluxes in all other filters. The uncertainties of the computed delays were determined via the model-independent Monte-Carlo simulations of the light curve using the FR/RSS method. For each simulated light curve the delay was computed both at the peak of the $\mathrm{CCF}\left(\tau_{\text {peak }}\right)$ and at the centroid of the CCF $\left(\tau_{\text {cent }}\right)$. The latter was determined at a level higher than $r>0.83 r_{\max }$. A total of 2000 Monte-Carlo realizations were considered. The $\tau_{\text {peak }}$ and $\tau_{\text {cent }}$ distributions were derived and were used to obtain $\pm 1 \sigma$ errors. Results of the CCF analysis are shown in Fig. 7 and given in Table 5. One can see in Fig. 7 that there is a skewness of the CCF curves towards the side of positive delays. This skewness increases with increasing wavelength, i.e. there is a tendency for the delay to be greater for longer wavelengths, though the uncertainties are large. As it was shown by Sergeev et al. (2005), this effect is present in twelve more Seyfert galaxies discussed in that paper. Sergeev et al. (2005) considered a model explaining these delays as a light travel effect. In that model, the optical emission is mainly re-procesed emission which arises in an accretion disk heated by an X-ray source above the disk. In this case the delay must reflect the geometrical size of the continuum emission region. 


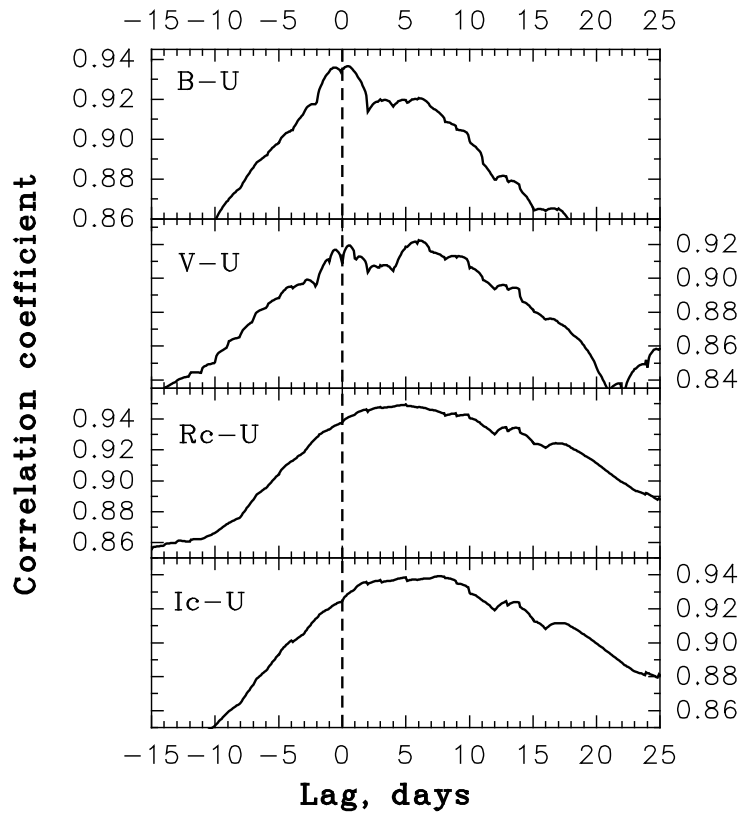

Fig. 7. Cross-correlation functions based on $U B V R \mathrm{c} I \mathrm{c}$ data.

Table 5. Cross-correlation results.

\begin{tabular}{lccccccc}
\hline \hline Bands & $\begin{array}{c}\tau_{\text {cent }} \\
\text { days }\end{array}$ & \multicolumn{2}{c}{ $\pm 1 \sigma$} & $\begin{array}{c}\tau_{\text {peak }} \\
\text { interval }\end{array}$ & \multicolumn{2}{c}{$\begin{array}{c} \pm 1 \sigma \\
\text { days }\end{array}$} & \multicolumn{2}{c}{ interval } \\
& int & \\
\hline$B / U$ & 2.2 & -3.4 & +6.8 & 0.4 & -0.8 & +3.3 & 0.94 \\
$V / U$ & 4.5 & -1.0 & +14.5 & 6.0 & -0.9 & +8.8 & 0.92 \\
$R \mathrm{c} / U$ & 8.3 & -2.5 & +15.1 & 5.0 & +2.1 & +12.1 & 0.95 \\
$I \mathrm{c} / U$ & 7.6 & -2.9 & +15.7 & 7.6 & +1.9 & +11.2 & 0.94 \\
\hline
\end{tabular}

\section{Summary}

The analysis of the most complete $U, B, V, R \mathrm{c}, I \mathrm{c}$ data for Mrk 335 obtained in 1995-2004 has shown that:

- Mrk 335 has a large amplitude of variability reaching about $1.1,0.9,0.7,0.3$ and $0.3 \mathrm{mag}$ in the $U, B, V, R \mathrm{c}$ and Ic bands, respectively. From the end of 1995 the brightness decreased systematically, and the variations of different amplitudes and duration are superimposed. Such variations of brightness are present in most Sy 1 galaxies.

- We also constructed a more complete light curve for Mrk 335, fitting the spectral continuum fluxes near $\lambda 5100 \AA$ (published by Kassebaum et al. 1997; Peterson et al. 1998a) to our broad-band data in the $V$ filter. Thus, we obtained a composite light curve for Mrk 335 covering a time interval of more than 15 years.

- The spectral energy distribution of Mrk 335 in optical wavelengths, in spite of the small excess in the $U$ and $R \mathrm{c}, I \mathrm{c}$ bands, is the same as for other Sy1 galaxies.

- The structure function analysis of Mrk 335 has shown that the variability of Mrk 335 can be caused by stochastic superposition of independent flares in a wide range of durations, the longest of which has a duration of about several hundred days. A slope of the power-law portion of the $S F$ is $b \sim 0.6-0.7$ and it is more consistent with the estimates for disk instability models. The estimate of the extension of the instability zone in an accretion disk (about $300 R_{\mathrm{S}}$ ) is also evidence that the instability in an accretion disk is able to explain the long-term optical variability.

- We also suspect the presence of a lag of some days in flux variations in the $V, R, I$ bands relatively to the $U$ band, that shows a tendency to be systematically higher for the $R c, I c$ bands than for $B, V$ ones. In the framework of the model discussed by Sergeev et al. (2005), where the optical emission is re-processed by an accretion disk heated by an X-ray source above the disk, such a delay can reflect the geometrical size of the continuum emission region.

Acknowledgements. We are grateful to our anonymous referee for many helpful remarks which led to an improvement of the manuscript. This work was supported by grant CRDF (US Civilian Research \& Development Foundation) No. UP1-2549-CR-03 and by RFBR (Russian Foundation for Basic Research) No. 03-02-17123.

\section{References}

Aretxaga, I., \& Terlevich, R. 1994, MNRAS, 269, 462

Bachev, R., Strigachev, A., \& Dimitrov, V. 2000, A\&AS, 147, 175

Bianchi, S., Matt, G., Haardt, F., et al. 1999, A\&A, 376, 77

Bohlin, R. C., Savage, B. D., \& Drake, J. F. 1978, ApJ, 224, 132

Boller, Th., Brandt, W. N., \& Fink, H. 1996, A\&A, 305, 53

Cid Fernandes, R., Sodre, L. Jr., \& Vieira da Silva, L. Jr. 2000, ApJ, 544,123

Collier, S., \& Peterson, B. M. 2001, ApJ, 555, 775

Courvoisier, T. J.-L., Paltani, S., \& Walter, R. 1996, A\&A, 308, L17

Czerny, B., Schwarzenberg-Czerny, A., \& Loska, Z. 1999, MNRAS, 303, 148

Czerny, B. 2005, Report on the Crimean AGN variability meeting, AGN variability from X-ray to radio, that was held in Crimea on June, 14-17, 2004, ASP Conf. Ser., ed. C. M. Gaskell, I. M. McHardy, B. M. Peterson, \& S. G. Sergeev, in press

Doroshenko, V. T., Sergeev, S. G., Merkulova, N. I., et al. 2005, Astrofizika, in press

Edelson, R. A., \& Malkan, M. A. 1986, ApJ, 308, 59

Gondoin, P., Orr, A., Lumb, D., \& Santos-Lleo, M. 2002, A\&A, 388, 74

González-Pérez, J. N., Kidger, M. R., \& Martín-Luis, F. 2001, AJ, 122,2055

Haardt, F., Maraschi, L., \& Ghisellini, G. 1994, ApJ, 432, L95

Kassebaum, T. M., Peterson, B. M., Wanders, I., et al. 1997, ApJ, 475, 106

Kawaguchi, T., Mineshige, S., Umemura, M., \& Turner, E. L. 1998, ApJ, 504, 671

Kolmogorov, A. N. 1941, Dokl. Acad. Nauk SSSR, 30, 229

Lyuty, V. M. 1971, Soobschenia of GAISh MSU, No. 172, 30

Osterbrock, D. E., \& Pogge, R. W. 1985, ApJ, 297, 166

Peterson, B. M., Wanders, I., Bertram, R., et al. 1998a, ApJ, 501, 82

Peterson, B. M., Wanders, I., Horne, K., et al. 1998b, PASP, 110, 660

Sergeev, S. G. 1999, Ph.D. Thesis, Crimean Astrophysical Observatory, Ukraine

Sergeev, S. G., Doroshenko, V. T., Golubinsky, Yu. V., et al. 2005, ApJ, 622, 129

Simonetti, J. H., Cordes, J. M., \& Heeschen, D. S. 1985, ApJ, 296, 46

Turner, T. J., \& Pounds, K. A. 1988, MNRAS, 232, 463

Turner, T. J., Nandra, K., Zdziarski, A. A., et al. 1993, ApJ, 407, 556

Veron-Cetty, M.-P., Veron, P., \& Gonçalves, A. C. 2001, A\&A, 372, 730

Wandel, A., Peterson, B. M., \& Malkan, M. A. 1999, ApJ, 526, 579

Winkler, H. 1997, MNRAS, 292, 273

Zheng, W., Kriss, G. A., Davidsen, A. F., et al. 1995, ApJ, 444, 632 